\title{
Is There a Link between Clinical Manifestation of Gastric Anisakiasis and Helicobacter pylori Infection?
}

Gastric anisakiasis is a parasitic disease caused by the consumption of seafood infested with third-stage larvae of the nematode Anisakis simplex or related species. Patients typically present with acute and severe abdominal pain, nausea, and vomiting. Endoscopic removal is the standard therapy. With the increase in incidence of gastric anisakiasis in the West, this disease has become widely recognized worldwide. ${ }^{1,2}$

Clinical records of gastric anisakiasis cases diagnosed between April 2003 and May 2017 at St. Luke's International Hospital (Tokyo, Japan), a tertiary referral center, were retrospectively reviewed. A total of 134 patients (54\% men; mean age, 41.8 years) were diagnosed with symptomatic gastric anisakiasis, with subsequent endoscopic removal of the larvae during this period. We reviewed the presence of atrophic gastric mucosa using the modified Kimura-Takemoto classification. ${ }^{3}$ Interestingly, $83.6 \%(112 / 134)$ of the patients had no atrophy, 10.4\% (14/134) had limited atrophy (antral and antral-predominant atrophy: C-1, C-2), and only 5.9\% had extensive atrophy (corpus-predominant and pan-atrophy: C-3, O-1, O-2, O-3). The prevalence of a non-atrophic stomach seemed high, considering the overall prevalence of Helicobacter pylori infection in Japan is approximately $40 \%{ }^{4}$ we therefore assumed that a significant number of patients had asymptomatic parasitic infection.

Although factors associated with the development of symptomatic gastric anisakiasis remain unknown, the correlation between clinical symptoms and gastric atrophy may be significant. A previous study analyzing 45 Anisakis specimens from 35 patients revealed that larvae penetrated normal mucosa more frequently than atrophic mucosa, and that patients had a significantly higher risk of developing clinical symptoms with infection in normal mucosa. ${ }^{5}$ We speculate that the clinical manifestations of gastric anisakiasis may be inversely related to $H$. pylori infection, given that atrophic gastritis is mostly due to H. pylori infection. Despite the possibility of selection bias in this report, further investigation is warranted to help clarify the mechanisms involved in the immune response to Anisakis larvae. With a decrease in prevalence of $H$. pylori infection among the younger population in East Asia, gastric anisakiasis may become more common.

\section{Conflicts of Interest}

The authors have no financial conflicts of interest.

\section{Yuto Shimamura ${ }^{1,2}$, Hirokazu Honda ${ }^{2}$ and Katsuyuki Fukuda ${ }^{2}$ Division of Gastroenterology, ${ }^{1}$ St. Michael's Hospital, University of Toronto, Toronto, Canada, ${ }^{2}$ St. Luke's International Hospital, St. Luke's International University, Tokyo, Japan}

\section{REFERENCES}

1. Audicana MT, Kennedy MW. Anisakis simplex: from obscure infectious worm to inducer of immune hypersensitivity. Clin Microbiol Rev 2008;21:360-379.

2. Hochberg NS, Hamer DH. Anisakidosis: perils of the deep. Clin Infect Dis 2010;51:806-812.

3. Kono S, Gotoda T, Yoshida S, et al. Can endoscopic atrophy predict histological atrophy? Historical study in United Kingdom and Japan. World J Gastroenterol 2015;21:13113-13123.

4. Ueda J, Gosho M, Inui Y, et al. Prevalence of Helicobacter pylori infection by birth year and geographic area in Japan. Helicobacter 2014;19:105-110.

5. Arai T, Akao N, Seki T, et al. Molecular genotyping of anisakis larvae in Middle Eastern Japan and endoscopic evidence for preferential penetration of normal over atrophic mucosa. PLoS One 2014;9:e89188.

Received: June 7, 2017 Revised: July 14, 2017 Accepted: July 14, 2017

Correspondence: Yuto Shimamura

Division of Gastroenterology, St. Michael's Hospital, University of Toronto, 30 Bond St. Toronto, ON M5B 1W8, Canada

Tel: +1-416-360-4000, Fax: +1-416-360-4000, E-mail: yutoshimamura1219@gmail.com

(cc) This is an Open Access article distributed under the terms of the Creative Commons Attribution Non-Commercial License (http://creativecommons.org/licenses/by$\mathrm{nc} / 3.0$ ) which permits unrestricted non-commercial use, distribution, and reproduction in any medium, provided the original work is properly cited. 